\title{
Dynamical properties of inertial confinement fusion plasmas
}

\author{
Kodanova S.K. * ${ }^{1}$, Ramazanov T.S. ${ }^{1}$, Issanova M.K. ${ }^{1}$ \\ ${ }^{1}$ IETP, Al-Farabi Kazakh National University, Almaty, 050040, Kazakhstan \\ *Corresponding author: kodanova@physics.kz
}

Study of the interaction processes of ion beams with dense plasmas is one of the important problems in the physics of inertial confinement fusion (ICF), warm dense matter and highpower lasers physics. Nowadays, a state of matter under extremely high pressure is an object of high interest. Investigation of dense matter has crucial importance for application [1] as well as for understanding of universe evolution. Particularly, many expectations associated with an ICF, where dense plasma is a working substance. The goal of ICF implosions is to achieve sufficiently high temperature and compression within deuterium-tritium (DT) fuel to sustain a thermonuclear burn [2].

One of the important values that describes the energy loss of the ions in plasma is the Coulomb logarithm. The Coulomb logarithm is obtained on the basis of effective potentials [3]. These interaction potentials take into consideration long-range many particle screening effects as well as short-range quantum-mechanical effects [4-7]. We investigate the energy loss of heavy particles in DT plasma and other energetic characteristics of fuel. Calculations of ions energy losses in the plasma for different values of the temperature and plasma density were carried out. Also, a comparison of the calculated data of ion stopping power with experimental and theoretical results of other authors was done.

\section{References}

[1] V.E. Fortov. Extreme states of matter on Earth and in the Cosmos. - Springer, (2009)

[2] N.A. Tahir et al. 2014 Phys. Rev. ST Accel. Beams 17, P.041003

[3] C.A. Ordonez, M.I. Molina. 1994 Phys. Plasmas, 1, 2515

[4] T. S. Ramazanov, S.K. Kodanova, Zh. A. Moldabekov, and M. K. Issanova. 2013 Phys. Plasmas, 20, 112702 .

[5] T. S. Ramazanov, Zh. A. Moldabekov, and M. T. Gabdullin. 2015 Phys. Rev. E., 92, 023104

[6] S. K. Kodanova, T. S. Ramazanov, M. K. Issanova, G. N. Nigmetova, and Zh. A. Moldabekov. 2015 Contrib. Plasma Phys. 55, 271

[7] M.K. Issanova, S.K. Kodanova, T.S. Ramazanov, and D.H.H. Hoffmann. 2016 Contrib. Plasma Phys. 56, No. 3-4, DOI 10.1002/ctpp.201500134 